\title{
SONIFYING THE SOLAR SYSTEM
}

\author{
Michael Quinton, Iain McGregor and David Benyon
}

\author{
Edinburgh Napier University, School of Computing, \\ Merchiston Campus, 10 Colinton Road, Edinburgh, \\ EH10 5DT, United Kingdom \\ \{m.quinton, i.mcgregor, d.benyon\}@napier.ac.uk
}

\begin{abstract}
Sound is potentially an effective way of analysing data and it is possible to simultaneously interpret layers of sounds and identify changes. Multiple attempts to use sound with scientific data have been made, with varying levels of success. On many occasions this was done without including the end user during the development. In this study a sonified model of the 8 planets of our solar system was built and tested using an end user approach. The sonification was created for the Esplora Planetarium, which is currently being constructed in Malta. The data requirements were gathered from a member of the planetarium staff, and 12 end users, as well as the planetarium representative tested the sonification. The results suggest that listeners were able to discern various planetary characteristics without requiring any additional information. Three out of eight sound design parameters did not represent characteristics successfully. These issues have been identified and further development will be conducted in order to improve the model.
\end{abstract}

\section{INTRODUCTION}

Sonification of Scientific Data has gradually become more established since 1985 [01]. The large amounts of data that make up the basis of cosmic research suggest that sonification may be a suitable tool for data analysis, where the technique has been used to an increasing extent. For members of the general public spectacular images of the planets and other space related phenomena could potentially be sonically enhanced to include additional data not easily conveyed through traditional imagery. These sonic signatures might not only increase the entertainment value, but also help educationally. It may also improve accessibility, by providing a richer experience for those who are visually impaired. Sonification acts like a sound effect in a film where it enhances or indicates the nature of a particular parameter. For example if taking a scene from a film where the sound of a steam engine is heard but is not represented visually on screen then the viewer still feels that there is a steam engine in that particular scene and can relate to it.

\subsection{Sonification and Planetariums}

Scientists, composers and sound artists have explored and implemented sonification in planetariums usually in the form of installations, exhibits or performances based on musical compositions. These sonifications offered an educational and entertainment value to the audiences.
Through sonification, abstract concepts like planetary movements, can be made more tangible and comprehensible to the general public. Since many sonifications have been created as artistic pieces a certain degree of scepticism from scientists has made them wary of using sonification as a scientific tool. Barrass [2] refers to the conflict between the more traditional scientific view with relation to data analysis and the new view, which embraces the advantages of the human auditory system and its cultural significance. There is a fine line between sonification as a means of scientific exploration and merely being perceived as a popular mass media marketing tool.

Out of the 58 examples of sonification that have been mentioned by Dubus and Bresin [3] the majority of these are related to scientific applications. In this list of examples it is interesting to note that only three sonifications related to astronomical sciences are mentioned. When compared to other areas of scientific study sonification ranks as one of the poorer fields of science. Considering the large amounts of data involved in astrophysics the use of sonification in this field can be explored in more detail. The difficulty arises when trying to find the right balance between artistic input and sonifications that are usable in scientific data analysis. It is for this reason that an end user approach was adopted for this study.

\subsection{Sonification}

The multidimensional and multidirectional nature of hearing, or the spherical nature of sound as described by Sterne [4], allows for a number of characteristics of sonified data to be recognised. By listening to data it is possible to perceive patterns and structures that may not be apparent using visual methods. Certain sounds can be relegated to the background and given lower priority allowing the user to carry out other tasks while listening. There is also the ability to filter out certain sounds in order to be able to focus on specific sounds within a dataset [5]. By intentionally not discerning individual sounds, complex sounds can be perceived as a whole allowing the listener to hear multiple audio streams in parallel. Sensitivity to high temporal and high frequency resolution makes us distinctly sensitive to rhythm and pitch allowing listeners to distinguish minute changes in details and enables the use of complex datasets. From slight changes in sound, users can detect a variation in data through which the listener can convey the parameters affected. Apart from being able to handle other tasks while listening to data, there is also the advantage that it is possible to listen to data without having to look at a screen or to even be seated in front it which is ideal for distance monitoring [6]. Hermann and Hunt [7] mention how humans are capable of identifying sound 
sources, spoken words and melodies under noisy conditions. Sound can be a tool for navigation in a fixed image as it portrays movement and offers spatiality. Time dimension is also well supported by sound mapping. Sound can be a potent means of allowing users to create mental image associations and this in turn strengthens memory [8]. It is also advantageous from the point of view of data storage where a single channel of uncompressed $640 \times 480$ video equals 200 channels of CD quality audio [9].

\subsection{Hearing in relation to Sonification}

Considering the sophistication of human hearing, sound can potentially be utilised effectively in the process of data analysis. Sound parameters can be attributed to data in order to represent various characteristics. In Table 1 basic elements of hearing as described by Levitin [10], are compared to sonification mappings that were used in a number of sonification projects that have been listed and categorised by Dubus and Bresin, [3]. It is worth noting that the sonification mappings can vary accordingly and are not necessarily arbitrary, and it is often the sound designer who designates the various parameters according to need. Dubus and Bresin have managed to show that the majority of projects used certain mappings related to specific parameters consistently.

The above mentioned sonification mapping parameters have been used in the sonification process of cosmic data, such as the sonification of Kepler space telescope star data [11] where the brightness values of certain stars were observed over long periods of time. Fluctuations in the brightness values indicated that planets were passing between the Kepler telescope and the star that was being observed. In order to sonify the data the software 'Sonification Sandbox' was used. 'Sandbox' is multipurpose software used for sonifying data. It allows users to map data to multiple auditory parameters such as timbre, pitch, volume and pan.

Table 1: - The relationship between hearing and sonification mapping possibilities

\begin{tabular}{|c|c|}
\hline Parameter & Sonification Mapping \\
\hline Loudness & Proximity, size, importance, energy \\
\hline Pitch & $\begin{array}{c}\text { Location, size, orientation, velocity, } \\
\text { motion, size, distinction }\end{array}$ \\
\hline Contour & $\begin{array}{r}\text { This would represent the overall } \\
\text { sonification }\end{array}$ \\
\hline Rhythm & Intensity, density, speed \\
\hline Tempo & Velocity, event rate \\
\hline Timbre & Proximity, intensity, importance \\
\hline Reverberation & $\begin{array}{r}\text { Motion, location, proximity, } \\
\text { spatialization }\end{array}$ \\
\hline
\end{tabular}

Sources: Levitin, [10] and Dubus \& Bresin, [3]

One of the most commonly used techniques of sonification in Astronomy is Audification. NASA has published numerous examples of audification, such as those from waves captured by the Cassini spacecraft as it travelled through our solar system. There are various examples of different solar system phenomena like solar winds, sounds from Jupiter, Saturn, the moons of Titan, Encaledus, the rings of Saturn and the Voyager 1 recordings from outside the heliosphere of our solar system (The heliosphere is the sun's magnetic field inflated to gargantuan proportions by solar winds [12]). These radio waves have been transposed by software platforms such as xSonify and brought into the human range of hearing [13]. Data like these can be immediately transposed in pitch. For example, readings from Jupiter of radio-astronomy data led to the discovery of whistlers, hiss and chorus.

\subsection{Sonification of Astronomical Phenomena}

Dubus and Bresin [3] describe how scientists are becoming more accustomed to using sonification as an analysis tool and that the community of researchers using this new tool has grown substantially. Out of the few sonifications made for space physicists none have been designed and tested with the end user [14]. This results in the sonifications being inadequate for the task of data representation and end up not being used. Dubus and Bresin [3] make reference to comments by Scaletti [15] where she states that sound attributed to data can only be called sonification once it has been done with the intent of understanding or communicating something about the original information.

The use of sonification in discerning space data is growing but not a new field. Donald Gurnett has been sonifying data from spacecraft for decades [16]. It was believed that space was a vacuum and therefore sound was unable to travel through it [17] but it has now been discovered that space is not a total vacuum and that stuff does exist between the stars at very low densities and pressures which makes the sound waves inaudible, but sound waves can actually travel through space [18]. This argument is further supported by Professor Carolin Crawford [19] and in a presentation entitled 'The Sounds of the Universe' she states that, "sound can be used as a diagnostic of cosmic phenomena, indirectly tracing the behaviour of astronomical objects: - whether the presence of lightning on Jupiter, or the physical structure inside distant stars."

Crawford [19] argues that sound is an effective means of illustrating certain aspects of astronomy, in particular radio signals. She also referred to the capability of sound to transfer significant amounts of energy across vast volumes of space. During this presentation Professor Crawford plays numerous examples of "sounds from space" but emphasizes the fact that hardly any of the sounds played during her lecture are the actual sounds recorded from space. Some of the sounds never existed. They are conversions of natural radio signals, which are part of the electromagnetic spectrum, into sound. For example, a recording of radio transmissions from the sun have to be speeded up 42,000 times from $0.1 \mathrm{~Hz}$ to $4.2 \mathrm{KHz}$ in order for them to become audible.

One aspect of space science that has grown popular recently is that of planet hunting. There are a myriad of exoplanetary systems where thousands of planets are being found, over 5000 found to date and 1800 confirmed as planets [20], [21]. The model of the solar system alone is already a rich and vast playground of possibilities and overwhelming amounts of data. In order to plough through this vast sea of information an efficient and effective means of data analysis has to be utilised. The use of sound could not only facilitate this process and reduce the amount of 
time needed to make these analyses, but also allows the listener to create mental images [8] and transport users onto for example, planetary surfaces, nebulae clouds and black holes.

By building an effective sonification of the solar system it can act as a prototype for building sonifications for exosolar planetary systems. The Planetarium model not only works as an educational tool for people who are not familiar with astronomy but it also acts as an indication that if people who are not familiar with the space sciences can discern what the sonification parameters represent then this would mean that a sonification designed for astro scientists using an end user approach may have more positive results.

\section{METHOD}

\subsection{Participants}

In order to design the sonification of the planets the user, in this case a planetarium representative, was involved in the data gathering process. An interview was conducted with a trained scientist and teacher.

For the testing of the sonification 11 people from the general public were interviewed, together with the representative from the planetarium. For the experiment 9 males and 3 females participated in the experiment with ages ranging from 24 to 56 .

\subsection{Materials}

The data gathering interview was part multiple choice and part interview, where further questions were asked about the parameters addressed through multiple choice. Audio recordings were made of the interview to be later transcribed.

The testing of the sonified model took place at various locations, as no central meeting place could be found, and to better accommodate the participants' different schedules. The participants were sat down amidst four speakers, two in front and two behind their heads, at close proximity. The choice of four speakers was determined by the fact that the planetarium would be using 5.1 surround system where surround sound would only be working on a flat plain with no up and down movement. Volume levels were kept within a safe range below 85dBA SPL (Peak) and were controlled by the sound designer. The sound designer triggered and manipulated sound live from a DAW using a MIDI controller. The participants were given a questionnaire; the first 4 sections were multiple-choice questions on which the respondents marked their choices, whereas the fifth section contained open-ended interview style questions, which were audio recorded and transcribed.

\subsection{Design}

Questions for the data-gathering interview were based on information found on a planetary fact sheet by NASA [22]. The resultant sonified model would be a sonic representation of 8 planets and their orbital revolutions around the listener who would be situated in the position of the Sun.

Other questions were also added to the interview. Physical properties such as rock, gas, ice, liquid, metal and fire were included. These elements would be used in order to distinguish between one planet and another. The model would be represented on a 4 channel surround system working on a flat plane configuration (no up and down movements) and would be working as an audio visual presentation.

Questions that related to the mechanics of the model were also included. The model could be speeded up or slowed down so that planets that revolve around the sun either at very slow or at fast speeds could be regulated. There was also the idea of being able to 'zoom in and zoom out' to a specific planet. This would mean that the planet would be brought closer to the listener by turning up the volume and making the timbre much brighter to replicate the impression that closer objects are louder and clearer than further objects. Maasø [23] describes this phenomenon in relation to the human voice and its relation to the three acoustic characteristics related to distance i.e. volume, timbre and reverberation. He referred to how listening is more precise on a horizontal level but less accurate at estimating distance. Listeners have difficulties distinguishing whether a sound is coming from 7 or 8 feet away but it can easily distinguish between sounds that are 9 inches or 9 feet away. By adjusting the three acoustic characteristics mentioned it is possible to suggest a sense of distance.

\subsection{Procedure}

In Section 1 the planetarium representative was asked to grade the importance of each parameter. The grading was based on a five-point scale running from not important (1) through to very important (5). Parameters that were graded 1 or 2 were left out of the model. These parameters were Orbital eccentricity, planet surface pressure, the global magnetic field of a planet, perihelion and aphelion and rings of a planet. An exception was made in relation to Saturn's rings that are the planets most distinct characteristic. Planetary rings had not qualified as an inclusive parameter. Section 2 clarified which of the parameters graded at score 3 were actually important to the planetarium since the questions delved into more detail. Parameters that scored 4 or 5 were to be included. Some of the parameters that were given importance for the model were not direct parameters that could be sonically represented that easily. For example, diameter could not be directly represented as a sonification parameter. It would have to be represented as the size of the planet in the sound design through pitch. A total of 17 questions out of the 19 attributes mentioned in the NASA planetary fact sheet were included in this section. Five parameters were excluded from the final model and 12 parameters were included.

In the case of the testing of the model participants had to answer a number of multiple choice questions which were designed in order to identify whether users were able to discern what the sonification was representing and to grade accordingly. They were not told which planets they would be listening to. In Section 1 part i participants were asked about the planet Mercury, Section 1 part ii Venus, Section 2 Earth and Mars combined and Section 3 Jupiter, Saturn, Uranus and Neptune combined. 


\subsection{Development of Model}

\subsubsection{Parameter Mapping}

Pitch was used to reflect the size of the planets. The scale ran between the notes $\mathrm{C} 4$ for Mercury (the smallest planet) and $\mathrm{C} 2$ Jupiter (the largest planet) the other planets were designated as follows: - Venus B3, Earth G3, Mars A3, Saturn D2, Uranus E2 and Neptune F2.

Sound design elements were used to represent atmospheric conditions, temperatures, air pressure, climate conditions etc. of each planet [24] [25]. Virtual synthesizers were used to create the sounds in the sound design process, which gave more flexibility than samples.

Each planet was also assigned with a low pass filter on each channel of the DAW that could be controlled manually during the experiment by using a MIDI controller allowing parameters such as cut off on the low pass filter to be altered live. This allowed the timbre to be manipulated during testing. Volume control was also controlled manually. The reason for not automating these parameters was so that the sound designer could create the zoom effect during the experiment.

Rhythm was linked to pulses representing the radio waves that are emitted by the planets. Tempo was related to the ability to speed up and slow down the model. This was not given importance by the representative of the planetarium. By speeding or slowing down the tempo one can hear faster planets more clearly and understand their orbits with more appreciation. A case in point is Mercury which only takes 88 days to orbit the Sun. On the other hand, at normal speed the very slow planets such as Neptune only get to revolve once around the surround sound system and therefore had to be speeded up so that the listener could hear the orbit of Neptune a couple of times around the sun. It takes Neptune approximately 165 years to orbit the sun once [24]. A visual model working on the same principle can be found at solarsystemscope.com [26].

A scale was created in order to represent the different speeds and celestial movements of the planets' orbits. When one observes various visual representations of the solar system it becomes evident that these representations are not according to scale. The number of orbits for each planet was worked out over 3 minutes running at a tempo of 5.33 seconds in a measure. Within the 3 minutes all 8 planets of the solar system would have at least looped around the sun once. Neptune the furthest of the planets only makes one revolution within this scale but this represents how slow Neptune actually is. In 2011 the planet completed one orbit since the date of its discovery [27]. Table 2 indicates how much time it takes each planet in order to make one revolution around the sun, and how these data have been sonified.
Table 2: Temporal Scale of planetary orbits [28]

\begin{tabular}{|c|c|c|}
\hline Planet & $\begin{array}{c}\text { Sonified time in } \\
\text { minutes }\end{array}$ & $\begin{array}{c}\text { Actual orbits in } \\
\text { Earth days/ months/ } \\
\text { years }\end{array}$ \\
\hline Mercury & 0.075 & 88 days \\
\hline Venus & 0.10 & 224.7 days \\
\hline Earth & 0.15 & 365 days \\
\hline Mars & 0.22 & 11.9 Years \\
\hline Jupiter & 0.43 & 29.7 Years \\
\hline Saturn & 1.05 & 84.3 Years \\
\hline Uranus & 1.53 & 164.8 Years \\
\hline Neptune & 2.55 & \\
\hline
\end{tabular}

The orbit was replicated by a surround panner which sent the sound through four outputs of a soundcard and was transmitted through a quadraphonic configuration. Every planet was automated so that it would move through the surround in accordance to the planet's speed.

The actual sonifications of each planet and of the solar system can be heard [29]: -

\section{RESULTS}

\subsection{Results from the Data Gathering Interview}

The parameters density, diameter of the planet, gravity, length of day of a planet, orbital period, mean temperature of the planet's surface and orbital velocity were given most importance by the planetarium representative (PR).

The parameters Mass of a planetary body, Distance from the Sun, Ability to zoom in and out on a planet, Atmospheric Characteristics were given less importance by PR but would still be included in the model.

Orbital eccentricity, planetary surface pressure, ring system, global magnetic field and Perihelion and Aphelion were to be excluded from the model.

There were 20 questions in section 2 and the replies were related to Timbre: - Closer planets are clearer than more distant ones (proximity), Rhythm/ Duration: Would represent the radio wave pulse emissions from planets, Tempo: - Variable speed of the planetary movements by altering the BPM in the DAW, Pitch: Smaller planets are higher in pitch than larger planets, Loudness: - Closer planets are louder than more distant ones (proximity), Reverberation: - Would represent distance.

\subsection{Results from Testing the Sonification Model}

\subsubsection{Interpretation of the characteristics of Mercury}

The first planet that the participants were asked to discern was information about the planet Mercury. The sonification of this planet was quite successful and participants were able to discern many of the planets characteristics. It is important to note that the listeners had no prior reference or 
baseline to which they could associate to or compare and they were not told which planet or planets they would be listening to throughout the experiment. Considering this factor participants were still able to discern the planets size, gravitational influence and atmosphere successfully. The planet was deemed by P2, P4, P5, P6, P7 and P8 as being of an average size and not a large one. P2, P6, P7, P8 and P9 discerned the gravitational pull as being of average strength. Almost all the participants except for P2 were able to discern Mercury's Magneto Sphere as one the planets main attributes and participants P1, P5, P6, P9, P10 and P11 were able to determine a lack of atmosphere or atmospheric conditions which is a precise discernment considering that Mercury does not have an atmosphere but instead has something called an exosphere made up of atoms which are blasted off its surface by solar radiation [27].

\subsubsection{Interpretation of the characteristics of Venus}

Venus was poorly represented sonically as a planet and listeners were not able to discern that many characteristics successfully. The only parameters that the participants were able to discern correctly was the strength of Venus's gravitational pull. P1, P2, P3, P4, P9 and P11 could also successfully discern that Venus was a larger planet than Mercury and that Venus is close to the sun (P3, P4, P5, P6, $\mathrm{P} 9, \mathrm{P} 10$, and P11). Participants scored poorly with regards to the planets type were Venus was designated as an Icy planet of cold temperatures and of mild atmospheric conditions (P1, P3, P4, P5, P6, P7, P8, P11 and PR). The sound design for a fiery planet like Venus was suggested by PR and was described as a 'chime-like popping' sound. It was probably the metallic properties of the sound that gave the impression of Venus being a cold icy planet. PR had also felt that the planet was of an average temperature like many of the other participants. This gives a further indication that the sound design for this planet is ineffective and has to be revised. Mars

\subsubsection{Interpretation of the characteristics of Earth \&}

The overall discernment between the two planets of Earth and Mars was fairly successful although there were slight problems with the sound design of Mars that had a negative effect on the results with regards to parameters concerning the planets' size. Participants P1, P3, P5, P7, P8, P10 and PR could successfully determine that both planets were close to each other and that there were slight differences in size between the two planets (P4, P6, P7 and P9). The only problem was that participants $\mathrm{P} 1, \mathrm{P} 2, \mathrm{P} 3, \mathrm{P} 6, \mathrm{P} 7, \mathrm{P} 8, \mathrm{P} 9$ and $\mathrm{P} 11$ mistakenly deemed Mars as being bigger than the Earth. With regards to characteristics P1, P4, P5, P6, P7, P9 and PR were able to determine that Earth had rocky and liquid characteristics but were unable to successfully discern Mars as being a Rocky planet except for PR who was the only candidate to designate Mars as being a rocky planet. The sound design of Mars gave the impression of being a larger and colder planet with ice qualities due to the horn like qualities that were used for the sound design. The metallic quality of the sound gave the impression of coldness and the depth of the sound gave the wrong impression of largeness. The sound design for the planet Mars would have to be revised.
3.2.4. Interpretation of the characteristics of Jupiter, Saturn, Uranus \& Neptune

In this section participants were asked to listen to the four planets of Jupiter, Saturn Uranus and Neptune simultaneously. The planets were introduced to the listeners one by one and then left to play at the same time for approximately three minutes. The results of this section were quite successful. This part of the listening experiment clearly indicated that P1, P2, P3, P5, P7, P8, P10, P11 and $\mathrm{PR}$ were able to hear different characteristics simultaneously and to be able to discern differences between the planets and recognize various characteristics from each planet. P1, P2, P3, P4, P5, P6, P10 and P11 found the experience of listening to four planets at the same time to be immersive. P2, P3, P5, P7, P8, P10, P11 and PR were able to distinguish the orbits of each planet clearly. Table 3 indicates the answers that participants gave for questions 4, 5 and 6 where for example in question 4 participants were asked to indicate how many planets they thoughts were either rock, ice, liquid, gas or fiery by writing down a number which would range from 1 to 4 . In Question 5 the listeners had to distinguish how many planets' were small, medium or large in size and in question 6 the participants had to work out the orbit speeds of the four planets by stating how many planets were orbiting at a fast, average or slow rate.

Table 3: Question 4 -6 Reported instances of parameters when comparing four planets

\begin{tabular}{|c|c|c|c|c|c|c|c|c|c|c|c|c|c|}
\hline \multicolumn{2}{|c|}{ Parameter } & $\bar{a}$ & $\Sigma$ & $\tilde{\Omega}$ & I & $\curvearrowleft$ & $\approx$ & $\hat{z}$ & 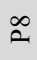 & ¿ & $\stackrel{\circ}{a}$ & $\bar{z}$ & $\stackrel{\approx}{2}$ \\
\hline \multirow{5}{*}{4} & Rock & 1 & 2 & 1 & 1 & 1 & 2 & 1 & 1 & 1 & 1 & & \\
\hline & Ice & 1 & 1 & & 1 & & 1 & & & 1 & 1 & 1 & \\
\hline & Liquid & 1 & & 1 & 2 & 1 & 1 & 1 & 1 & & 1 & & \\
\hline & Gas & 1 & 1 & 2 & & 1 & & 1 & 1 & & 1 & & 2 \\
\hline & Fire & & & & & 1 & & 1 & 1 & & 1 & 1 & 2 \\
\hline \multirow{3}{*}{5} & Small & & 1 & 1 & 2 & 1 & & 2 & 1 & & 1 & 2 & \\
\hline & Medium & 2 & 1 & 2 & 2 & 1 & 1 & 2 & 1 & 1 & 2 & 1 & 2 \\
\hline & Large & 2 & 2 & 1 & & 2 & 3 & & 2 & 1 & 1 & 1 & 2 \\
\hline \multirow{3}{*}{6} & Fast & 1 & 1 & 1 & 1 & 1 & 1 & & 1 & 1 & 1 & 2 & 2 \\
\hline & Average & 2 & 2 & 2 & 2 & 2 & 2 & 2 & 1 & 2 & 2 & 1 & \\
\hline & Slow & 1 & 1 & 1 & 1 & 1 & 1 & 2 & 2 & & 1 & 1 & 2 \\
\hline
\end{tabular}

\subsubsection{General interpretation of all eight planets playing} simultaneously

In this last part of the listening experiment the participants were asked to listen to all eight planets at the same time. The sound of each planet was triggered at the same time and was left to play for approximately three and a half minutes. In this section participants were asked more general questions related to main aesthetics. P2, P3, P4, P5, $\mathrm{P} 8, \mathrm{P} 10, \mathrm{P} 11$ and PR found that the soundscape was immersive and $\mathrm{P} 1, \mathrm{P} 6$ and $\mathrm{P} 9$ found that the sonification had a musical quality to it. P1, P3, P4, P5, P6, P9, P11 and $\mathrm{PR}$ found it to be harmonious and $\mathrm{P} 2$ and $\mathrm{P} 8$ found it familiar. There was only one participant, P7 that found the soundscape to be confusing. The listeners were then asked to determine whether or not they could follow differences 
in orbit speeds, planet size, proximity and climate. Table 4 indicates the scores for these parameters. From the table the parameter climate is the least one that listeners were able to distinguish due to all the different sounds that were happening at once. Proximity was the characteristic mostly discerned by the listeners where they were able to perceive planets that were closer and others that were further away. Finally the participants were asked to classify the quality of the sound design ranging from poor to good. Most of the participants found the sound design to be good. P7 and P10 found it to be fair while P9 graded it as not bad.

Table 4: Parameters that listeners were able to distinguish clearly

\begin{tabular}{|c|c|c|c|c|c|c|c|c|c|c|c|c|}
\hline Parameter & $\bar{z}$ & $\Sigma$ & $\tilde{\Omega}$ & $\Xi$ & $\curvearrowleft$ & 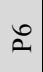 & $\hat{\mathbf{a}}$ & $\stackrel{\infty}{\infty}$ & ¿ & $\stackrel{\circ}{2}$ & $\bar{z}$ & 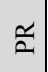 \\
\hline Orbit & • & • & & • & • & & • & • & & & • & \\
\hline Size & . & • & . & • & • & & • & - & & & & \\
\hline Speed & - & • & • & . & . & & - & - & • & & & . \\
\hline Proximity & • & . & . & & . & - & • & - & . & • & & . \\
\hline Climate & . & & & & . & & . & & & & . & . \\
\hline
\end{tabular}

\subsubsection{The Interview}

In question 1 participants were asked the reason for the rating they had given the model in section 4. The participants generally commented that they found the model to be immersive, it gave a good idea of spatiality, that the sounds of the planets were distinct but at the same time there was a balance between them, and that the sounds evoked images of planets and planetary landscapes.

In the second question the participants were asked to elaborate upon what they liked about the model. In general participants liked the distinction between the planets, and the amount and quality of the detail that were portrayed by the sounds. PR was impressed by the way that proximity was presented and how through immersion one felt that something was coming closer or moving away.

In question 3 participants were asked what they disliked about the model. There were certain resonances from one particular planet that did disturb some of the participants, P6 and P7 were particularly bothered by this. PR said that there were times when it got confusing. Rather than being an element of dislike it was more a matter of losing focus and not being able to discern the detail anymore.

Question four asked the participants how they would create their own sound design of the solar system. Many of the participants felt that they were unable to answer this question due to a lack of knowledge regarding the subject of sound design. Other participants such as P3, P4, P6, P7, $\mathrm{P} 10$ and $\mathrm{P} 11$ picked up on points relating to using the size and representing the dimensionality by using pitch or using smaller sounds for smaller planets and larger sounds for larger planets. PR replied the following:

“... I think you got it quite right according to my ideas and tastes..."

Question 5 asked participants whether they thought the sound design was an effective tool for representing data and question 6 asked whether sound design could enhance visual presentation of planets and whether the participants would use sound to represent the planets. All the participants including the PR agreed that sonification was an effective tool for representing data in one way or another and all felt that that sound would enhance the visual experience.

Only P10 disagreed that sound could be used as a scientific tool due to its subjective nature. P10 did agree that sound enhances the visual and could be used effectively to that extent. P8 and P11 felt that sonification helps to make scientific data accessible to the layman. This was a valid point especially when considered in the case of a planetarium where the users will not necessarily be knowledgeable about the planets. P3 made an interesting comment by stating that sound is a language that can be taught. Once sound has semantic value then discernment of data can be comprehended more easily by the listener and can be relayed to other listeners more effectively. P5, P9, P10 and PR found that sound acts as an effective memory tag and would also enhance visual memory much more. The general feeling was that sonification creates immersion that allows the listener to be drawn into the data and to share a more intuitive response to it.

\section{DISCUSSION}

\subsection{The Subjectivity of Sound}

In reference to Hegarty [30] where an isolated sound is important to guide the listener. If the listener is unable to create the suitable mental image through the sonification then it is ineffective.

Should sonification follow strict parameters where certain sounds always represent certain characteristics? This reminds us of Hermann's [31] comments that there were no specific guidelines determining how sonification is made. This remains to be a question of 'for and against' in the auditory display community. Let us take for example the high pitched and timbre sounds that seemed to evoke feelings of coldness in the participants of this experiment. There is an apparent trend in relation to sounds of this nature and the images they evoke in the listener. If further testing does indicate that the majority of participant's related high frequency sounds to a feeling of coldness, then this could become a standardised representation of this sensation. This makes the sonification reproducible, that the system can be used with different data and in repetition with the same data Hermann [31].

Although sound is subjective and everyone experiences it in their own way, there are common elements that work collectively [32]. There are various examples from the results that show common elements that the participants were able to discern. Idhe [33] emphasizes that the listener should be aware that one's beliefs will determine ones perspective of the sound and that the listener should listen to the sound itself. The beginning of the test might have induced a form of listening that searched for association since the null existence of one automatically caused the listeners to create an internal marking scale that they could relate to in order to be able to perceive the first planets that they listened to. As the test proceeded participants became more confident in their listening abilities. P10 had stated that the subjectivity of sound would not allow it to be an efficient scientific tool, but sound is measurable and it is 
mathematical making it an efficient tool for representing numerical data.

\subsection{Human Hearing vs. Parameter Mapping Sonification}

The parameters of hearing given by Levitin [10] and the sonification parameters mentioned by Dubus and Bresin [3] in relation to hearing are loudness, pitch, contour, rhythm, tempo, timbre and reverberation and the sonification mappings in relation to these parameters are compared.

Participants could discern particular parameters because these sounds were mapped in accordance with the way in which human hearing works. In the case of proximity participants scored high. When the planets were played individually they were brought closer or taken further away from the listener by reducing the timbre and amplitude.

In the case of rhythm, listeners were able to relate to the orbits of the planets and to distinguish between the different speeds that the different orbits were moving at in comparison to each other rather than in comparison to the sun. Elements of reverberation were not emphasized in the testing and participants were not asked about it in the questionnaire.

Contour would be related to the participant's ability to hear the model as a whole. Eight participants, including the planetarium representative, heard the model as harmonious and two others heard it to be familiar. These participants were able to hear the overall impression created by the sound of all eight planets playing and to take it in as one whole composition.

Pitch was calculated by participants in relation to each other. This meant that one planet always had to act as the baseline for the others to be compared to. When planets were played individually it was difficult to guess the size, especially in the case of the very first planet where there was nothing to compare it to.

Regarding the human hearing vs. parameter mapping of sonification when considering the positive results from the test and especially since the participants had no visual aid, guidance or even a comparative baseline then it can be concluded that the parameter mapping was successful.

\subsection{The End User Approach}

In this research and in research carried out by Diaz Merced et al. [14] no sonifications have been found that have been tested with the end user in the field of Space sciences. Diaz Merced et al. [14] have been designing the sonification software xSonify over the years and the final improvements made to the program have been made by involving the end user in the process.

There is one common aim in both studies that is clearly defined in the Diaz Merced et al. [14] report. The sonification must act as a common platform where blind and sighted people can be aware of the same events through the sonification and will be able to share the similar knowledge of what they have achieved through the sonification process.

One difference noted between the studies is that in the Merced et al. [14] report there is no mention of any spatial representation of the sonified data. Only volume, pitch and timbre have been mentioned as the parameters within which the sonification is made. The planetarium model gave importance to the spatial element. The spatial element helps to create immersion as was seen in the experiment carried out by Turner et al. [34] where a sense of a place was simulated in another place successfully. This is the level of immersion that can be achieved by using a surround configuration and also allows parameters to be mapped out more easily. For space data exploration this could be essential as multiple layers of data can be distributed in ways that make the data easier to listen to. Sterne [4] states that hearing immerses its subjects, it also places the listener inside an event and vision gives perspective.

\section{CONCLUSION}

It is interesting to note that this listening experiment was conducted on an audience of non-scientific people whose knowledge of astronomy is limited and yet they were able to discern a lot of detail from the model. The people were not involved in the sound design process itself. It is also not known how much knowledge the participants actually had with regards to the solar system. The planetary representative was able to determine characteristics more precisely because he was more knowledgeable about the subject and because he was involved in the sound design process too. If the lay person was able to determine so many details of the sound design without any prior guidance or knowledge of what they were listening to then this reflects that sonification is an effective means of representing data. With a couple of adjustments that would have to be made in order to address the problems with certain aspects of the sound design with regards to Venus and Mars then the Solar System model could then act as a comparative model for exosolar planetary systems. This is the same approach that is usually employed by scientists by comparing exosolar systems to our solar system in order to determine how these planetary systems work. If the sonification of a planetary system could be conducted with Astro scientists as the end user and where the sonification is specifically designed and mapped out according to their needs then they may be able to determine much more from such a sonification model and to be able to use it efficiently as a scientific tool. If the sonification is used with a visual component then the effectiveness of any solar planetary model will be enhanced.

As future work there are improvements that need to be made with regards to the sound design of certain planets. Once the sound design has been arranged testing can start again and a fresh batch of participants can be chosen in order to widen the sample to see what works and what does not and a more consistent sonified model of the solar system can be built and can also find use outside the planetarium market. The model of the solar system can act as a guideline or basis so that further sonifications for exosolar planetary systems can be built and can also be used as a comparative model against exo-solar systems. The work on exo-solar planetary systems will be aimed at Astro scientists that work in the field of exo-solar planetary science. The sonification can be used as a scientific tool which the scientists can use in order to analyse large portions of data and to find similar patterns or differences between the different systems. 


\section{REFERENCES}

[1] Hermann, T., \& Hunt, A. (2005). Guest editors' introduction: An introduction to interactive sonification. IEEE multimedia, (2), 20-24.

[2] Barrass, S. (2012). The aesthetic turn in sonification towards a social and cultural medium. AI \& society, 27(2), 177-181.

[3] Dubus, G., \& Bresin, R. (2013). A systematic review of mapping strategies for the sonification of physical quantities. PloS one, 8(12), e82491.

[4] Sterne.J., (2012). The sound studies reader. New York :Routledge,

[5] Lunn, P., \& Hunt, A. (2011). Listening to the invisible: Sonification as a tool for astronomical discovery.

[6] Vogt, K., de Campo, A., \& Eckel, G. (nd) An Introduction to Sonification and its Application to Theoretical Physics.

[7] Hermann, T., \& Hunt, A. (2011). The sonification handbook. Berlin: Logos Verlag.

[8] Minghim, R., \& Forrest, A. R. (1995, October). An illustrated analysis of sonification for scientific visualisation. In Proceedings of the 6th conference on Visualization'95 (p. 110). IEEE Computer Society.

[9] McGee, R. (2009), Auditory Displays and Sonification: Introduction and Overview.

[10] Levitin, D. J. (2011). This is your brain on music: Understanding a human obsession. Atlantic Books Ltd.

[11] Winton, R. J., Gable, T. M., Schuett, J., \& Walker, B. N. (2012). A sonification of Kepler space telescope star data.

[12] NASA/gov, 2014, accessed June 2015, http://science.nasa.gov/science-news/science-atnasa/2013/01nov ismsounds/

[13] NASA/ JPL, 2006, accessed June 2015 , http://saturn.jpl.nasa.gov/news/cassinifeatures/feature20060 $424 /$

[14] Diaz-merced, W.L., Brewster, S., Candey, R.M., Schneps, M. (2013) "A study of the use of a sonification prototype by astrophysicists". CHI'13, Paris, France

[15] Scaletti C (1994) Auditory display: sonification, audification and auditory interfaces, Addison Wesley Publishing Company, chapter 8: Sound synthesis algorithms for auditory data represen9tations. pp. 223-251.

[16] Feder, T., (2012) 'Shhhh. Listen to the data', Print edition,65,20-22, DOI:http://dx.doi.org/10.1063/PT.3.1550

[17] Blackstock, D. T. (2000). Fundamentals of physical acoustics. John Wiley \& Sons.

[18] O'Brien, T (2014) 'Sounds of Space', http://proftimobrien.com/2014/03/sounds-of-space/ , accessed April 2015

[19] Crawford, C. (2011) 'Sounds of the universe', Gresham College, accessed May 2015, http://www.gresham.ac.uk/lectures-and-events/the-soundsof-the-universe

[20] PlanetQuest (2015). 'Exo planets 2020', NASA Jet Propulsion Lab, accessed August 2015, http://planetquest.jpl.nasa.gov/news/208

[21] Exoplanets.org (nd), accessed August 2015, http://exoplanets.org/

[22] NASA, 2004, 'Planetary fact sheet', accessed July 2015,

http://nssdc.gsfc.nasa.gov/planetary/factsheet/planetfact not $\underline{\text { es.html }}$
[23] Maasø, A. (2008). The proxemics of the mediated voice. Lowering the boom: critical studies in film sound, 3650

[24] Space.com, 2015, accessed July 2015, http://www.space.com/

[25] NASA Solar System Exploration, 2015, accessed June 2015, https://solarsystem.nasa.gov/planets/index.cfm

[26] solarsystemscope.com, nd, accessed July 2015, http://www.solarsystemscope.com/

[27] Space.com, 2014, accessed July 2015, http://www.space.com/36-mercury-the-suns-closestplanetary-neighbor.html

[28] Universe Today, 2009, Last accessed July 2015, http://www.universetoday.com/37507/years-of-the-planets/ [29] Quinton, M (2016), Soundcloud, last accessed April 2016 ,

https://soundcloud.com/michael-quinton napier

[30] Hegarty, P., (2012) 'A Chronicle Condition: Noise \& Time', Chapter 1, Goddard, M., Halligan, B., \& Hegarty, P. (Eds.). (2012). Reverberations: The philosophy, aesthetics and politics of noise. Bloomsbury Publishing USA.

[31] Hermann, T. (2008). Taxonomy and definitions for sonification and auditory display.

[32] Chion, M. (1994). The three listening modes. AudioVision: Sound on Screen, 26-32. COMOS EU, (2013) 'From

$\mathrm{X}$ rays to music', accessed June 2015, http://www.fp7space.eu/Newsletter Archive/COSMOS+ Newsletter12.pdf [33] Ihde, Don. 1974. The Auditory Dimension. In Listening and Voice: A Phenomenology of Sound. Athens: Ohio University Press. Pp. 49-55, Sterne, J. (Ed.). (2012). The sound studies reader. Routledge.

[34] Turner, Phil. McGregor, Iain. Turner, Susan. Carroll, Fiona., 2003. "EVALUATING SOUNDSCAPES AS A MEANS OF CREATING A SENSE OF PLACE" (July): 69. 